\title{
A Scheme for Targeting Optical SETI Observations
}

\author{
Seth Shostak \\ SETI Institute, 2035 Landings Drive, Mountain View, CA 94043, \\ U.S.A. \\ Ray Villard \\ Space Telescope Science Institute, 3700 San Martin Drive, Baltimore, \\ $M D$ 21218, U.S.A.
}

\begin{abstract}
In optical SETI (OSETI) experiments, it is generally assumed that signals will be deliberate, narrowly targeted beacons sent by extraterrestrial societies to large numbers of candidate star systems. If this is so, then it may be unrealistic to expect a high duty cycle for the received signal. Ergo, an advantage accrues to any OSETI scheme that realistically suggests where and when to search. In this paper, we elaborate a proposal (Castellano, Doyle, \& McIntosh 2000) for selecting regions of sky for intensive optical SETI monitoring based on characteristics of our solar system that would be visible at great distance. This can enormously lessen the amount of sky that needs to be searched. In addition, this is an attractive approach for the transmitting society because it both increases the chances of reception and provides a large reduction in energy required. With good astrometric information, the transmitter need be no more powerful than an automobile tail light.
\end{abstract}

\section{Targeting of Optical Signals}

While interstellar radio beacons might or might not be deliberately targeted, it is generally assumed that optical beacons definitely would be. The reason for this is found in the energy cost per bit. It has been estimated (Ekers et al. 2002) that, while one photon is sufficient to convey a bit of information at optical wavelengths, in the radio regime a minimum of 40 photons is required. For tenmicron infrared light and $21 \mathrm{~cm}$ radio radiation respectively, this translates to a ratio of 500 in the energy per transmitted bit, clearly favoring the use of radio.

Optical signalling is expected to remedy this high energy cost by narrow focussing; for example, by forming a beam of $\sim 10 \mathrm{AU}$ centered on the targeted star. For various reasons, short $(\sim 1 \mathrm{~ns})$ pulses are the preferred optical signal type. Targets could be "pinged" sequentially, perhaps at a rate as high as 1 10 per second (Horowitz 1998). While this gives short (typically 100 to 1000 seconds) revisit times for a target list of 1000 star systems, the revisit time lengthens as the number of targets increases. If a transmitting civilization pings $10^{6}$ star systems with a single device, then the revisit time is about once per day, assuming a 0.1 second transmission. 
This is important given current strategies for optical SETI (OSETI). To date, most OSETI investigations have adopted the approach of the earliest radio SETI experiments by individually targeting nearby, mostly Sun-like stars. However, two-dimensional detectors will permit new experiments to operate in a survey mode, scanning the entire visible sky approximately once per year (Horowitz 1998). While surveys obviate the need to choose among targets, they are compromised by limited observing time on any particular object. This would decrease sensitivity for radio observations, but won't affect optical experiments as the integration time is set by the (presumed short) pulse length. However, OSETI sky surveys limit the type of signal that can be found to those with high duty cycles. The chance that a sky survey will find a civilization that pings $n$ star systems, including us, is clearly only one in $n$ for each scan of the sky. This could be a discouragingly small number, implying a long wait before success.

To vastly improve the chances for OSETI success, we elaborate a method of synchronization described by Castellano et al. (2000) that is both obvious to the transmitting civilization and to us. This is in the spirit of similar schemes proposed for radio SETI experiments that rely on supernovae or eclipsing binaries to provide a synchronization opportunity that tells us when to look, and where (Makovetskii 1977; Shostak 1997). The method depends on a phenomenon that is common enough to be interesting, sufficiently repetitive to be useful for a serially transmitted beacon, and allows for extraordinarily fine spatial targeting, thus greatly reducing transmitting costs.

\section{Transits as Synchronizers}

Planetary transits provide a useful way of discovering and investigating extrasolar worlds, particularly terrestrial-class planets that are too small to be detected by other search techniques. The gas-giant planet orbiting HD 209458, found using spectroscopic techniques, was also detected in transit (Henry et al. 1999). While HD 209458's planet is a so-called "hot Jupiter" (its orbital period is only four days), this technique - even when using ground-based telescopes - is capable of revealing Earth-size worlds. The Kepler space mission, scheduled for launch in 2007, will monitor $\sim 10^{5}$ stars for transits, and is expected to uncover hundreds of terrestrial planets.

In addition to being an easily detectable phenomenon, transits also afford the opportunity to judge a world's habitability. The transit time, coupled with knowledge of the star's size (from its spectral classification) gives the planet's orbit, and therefore the irradiance. But atmospheric spectral lines, as detected for example in the case of HD 209458 (Goss Levi 2002), could also implicate biology. This does not offer as good signal-to-noise as direct reflectance spectra, but is still a worthwhile, complementary experiment. Transits therefore bid us the possibility of both planetary detection and spectroscopic assessment of the atmospheres to look for the spectral fingerprints of a biosphere. It is reasonable to assume that extraterrestrial societies will have conducted similar surveys, and cataloged habitable planets for follow-up study.

Transits also provide an invaluable and obvious method for temporal synchronization between two civilizations many light-years apart. A transmitting society that sends an OSETI signal to a planet either during the transit as 
observed from the transmitter, or timed to arrive when the transit occurs in the targeting system, might encourage detection, since such times will be perceived as special occasions for the occupants of the transiting planet to look in the transmitter's direction. The ability of technological civilizations on both planets to clearly and unequivocally recognize the unique and precise geometric alignment and temporal "window of opportunity" makes this technique especially promising and robust. In addition, for planets in Earth-like orbits, these opportunities occur at intervals that are short enough to be interesting.

Synchronizing with transits requires either the transmitting or receiving party to accurately know the distance between the two star systems. Since the number of targets will be far smaller than the number of potential senders, the lesser burden for acquiring this information is on the transmitting end (and might be possibly concomitant to the discovery and evaluation of targets). Ergo, the reasonable strategy for receivers (us) is to search for signals now from star systems in the Sun's anti-direction, stars that are able to see us in transit, rather than trying to account for the propagation time to all possible senders.

\section{Target Areas}

The geometry of transit for a planet in approximately circular orbit of radius a defines a disk-like patch in the anti-Sun direction of angular size $\theta=2 r_{*} / a$, in which $r_{*}$ is the star's radius. For the Earth, $\theta \approx 0.5$ degree, which means that from random directions the chance of alignment permitting observation of a transit is $1 / 360$. The length of transit $\tau \sim r_{*} P / \pi a$, in which $P$ is the orbital period of the planet. For Earth, $\tau=0.5$ days.

Taking advantage of this synchronization scheme for OSETI is simple. One merely monitors either individual stars or the entire sky with the 0.5 degree patch on the ecliptic that corresponds to the anti-Sun direction. A single "light bucket" optical system is possible, although this will increase the background noise due to starlight. Two-dimensional detectors that segment the sky (such as will be used for the Harvard sky survey, Horowitz 1998), but are not necessarily aligned with individual stars, ameliorate this problem.

Clearly, not all positions along the ecliptic are equally desirable search locations. If there are $N$ contemporary galactic beacon transmitters, then the typical distance to the nearest of these able to see Earth in transit is as given in Table 1.

We see that for an assumed total number of contemporary technical societies $\leq 10^{6}$, we can just as well constrain our observations closer to the galactic plane, as indicated in the last column of the table. This argues for observing near the solstices, when the anti-Sun direction coincides with the position at which the ecliptic crosses the plane of the Milky Way. The optimal time to observe, assuming a million or less contemporary extraterrestrial societies in the Galaxy, is during the six week periods centered on these dates.

\section{Energy and Precision Considerations}

Suppose that 10 photons/pulse are required for detection (a number consistent with present-day experiments), and that the broadcaster is using the aforemen- 
Table 1. Stars Able to See Earth in Transit

\begin{tabular}{|l|l|l|l|}
\hline $\begin{array}{l}N-\text { Total } \\
\text { Number of } \\
\text { Transmit- } \\
\text { ting Galactic } \\
\text { Civilizations }\end{array}$ & $\begin{array}{l}\text { Number of } \\
\text { Civilizations } \\
\text { Able to See } \\
\text { Transit }\end{array}$ & $\begin{array}{l}\text { Distance to } \\
\text { Nearest Civi- } \\
\text { lization Able } \\
\text { to See Transit } \\
\text { (L-Y) }\end{array}$ & $\begin{array}{l}\text { Max. Galactic } \\
\text { Latitude of } \\
\text { Nearest Able } \\
\text { to See Transit }\end{array}$ \\
\hline $10^{8}$ & $310^{5}$ & 700 & $90^{\circ}$ \\
\hline $10^{6}$ & 3,000 & 3,000 & $18^{\circ}$ \\
\hline $10^{4}$ & 30 & 20,000 & $3^{\circ}$ \\
\hline $10^{2}$ & $<1$ & & \\
\hline \hline
\end{tabular}

Note: Half-thickness of stellar disk is taken to be 1000 l-y.

tioned scheme of beaming to a disk $10 \mathrm{AU}$ in diameter, centered on the targeted star. Our SETI telescope is assumed to have a $1 \mathrm{~m}^{2}$ collecting area. If the transmitted pulses consist of 10 micron infrared photons (to better penetrate interstellar dust at large distances), the required energy is $3.510^{5}$ Joules per pulse, or a power consumption of $35 \mathrm{MW}$, assuming a one second pinging signal containing 100 pulses. The transmitter mirror aperture required to target 10 $\mathrm{AU}$ from 1000 light-years is $63 \mathrm{~m}$ (this could, of course, be most easily achieved with an array of smaller mirrors.)

While the power requirements are independent of the distance between transmitter and target, they are nonetheless substantial. An interesting advantage of the transit scheme proposed here is the reduction in required beacon power since the targeted area need be no bigger than the star. In the case of a Sun-like star $\left(r_{*}=7.010^{8} \mathrm{~m}\right)$, this amounts to 0.08 Joules per pulse, or a power requirement (assuming the same 100 pulse per second ping) of a mere 8 watts, comparable to an automobile tail light! For comparison, Project Phoenix, the most sensitive of the current microwave radio SETI searches, could detect a 10 MW signal from $1000 \mathrm{l}-\mathrm{y}$, assuming a transmitting antenna $\sim 100 \mathrm{~m}$ diameter.

The small required power levels that can be used for the transit synchronization scheme come at a cost. High precision is required in target position (30 microarcseconds at $1000 \mathrm{l}-\mathrm{y})$, proper motion $(<0.02 \mathrm{~m} / \mathrm{sec}$ error $)$, and distance (one part in $10^{6}$ at $1000 \mathrm{l}-\mathrm{y}$ ). An interferometric optical system with maximum baseline of $14 \mathrm{~km}$ for the transmitter is also necessary to achieve the small beam sizes suggested here. While these accuracies are substantially beyond our own capabilities, there is no reason in principle why they could not be obtained with relatively straightforward space-based instrumentation. If such requirements prove either too stringent or too costly, one can raise the transmitter power to a still-modest 100 kilowatts, and broaden the transmitting beam to 100 times the star diameter. This relaxes the precision necessary for position and proper motion by two orders of magnitude, bringing them into a range that will be within our own capabilities in the near future. 


\section{Conclusion}

We have elaborated a scheme useful for synchronizing the transmission and reception of optical beacons, and one that does not require high-precision knowledge on the part of the receiver (us). This scheme offers four important advantages to OSETI experiments:

- By offering a logical time to expect signals, the enormous improbability of stumbling across an intermittent, serial optical beacon is greatly ameliorated. The scheme is so simple in concept, it should be apparent to extraterrestrial societies engaged in their own OSETI programs.

- Individual stars need not be searched, nor are full-sky surveys required or desirable. This greatly increases the efficiency and repeatability of such a search.

- Assuming that the number of contemporary galactic civilizations is less than a million, only the 36 degree path of the ecliptic centered at the point where it crosses the galactic plane $\left(6^{h} \mathrm{RA}\right.$ in the north, and $18^{h} \mathrm{RA}$ in the south) need be observed.

- The energy cost to the transmitting civilization is enormously reduced compared to targeting an entire inner solar system, thereby reasonably improving the chances that such optical beacons are deployed. The transmitter power varies inversely as the targeting capability of the extraterrestrial civilization. The civilization must also have a precise distant measurement to our star so that the signal arrives precisely on schedule.

\section{References}

Castellano, T., Doyle, L., \& McIntosh, D. 2000, in IAU Symp. 202 (IAU: Manchester), ref. Code E107.C

Ekers, R. D., Cullers, D. K., Billingham, J., \& Scheffer, L. K. 2002, in SETI 2020: A Roadmap for the Search for Extraterrestrial Intelligence, (SETI Press: Mountain View,CA)

Goss Levi, B. 2002, Physics Today, 55, 19

Henry, G., Marcy, G., Butler, P., \& Vogt, S. 1999, ApJ, 529, L41

Horowitz, P. 1998, The Technical Case for Optical and Infrared SETI, http:/seti. harvard.edu/oseti/ tech.pdf

Makovetskii, P. V. 1977, Soviet Astronomy, 21, 251

Shostak, G. S. 1997, in Astronomical and Biochemical Origins and the Search for Life in the Universe, ed. C.B. Cosmovici, S. Bowyer, \& D. Werthimer (Bologna: Editrice Compositori), 719 


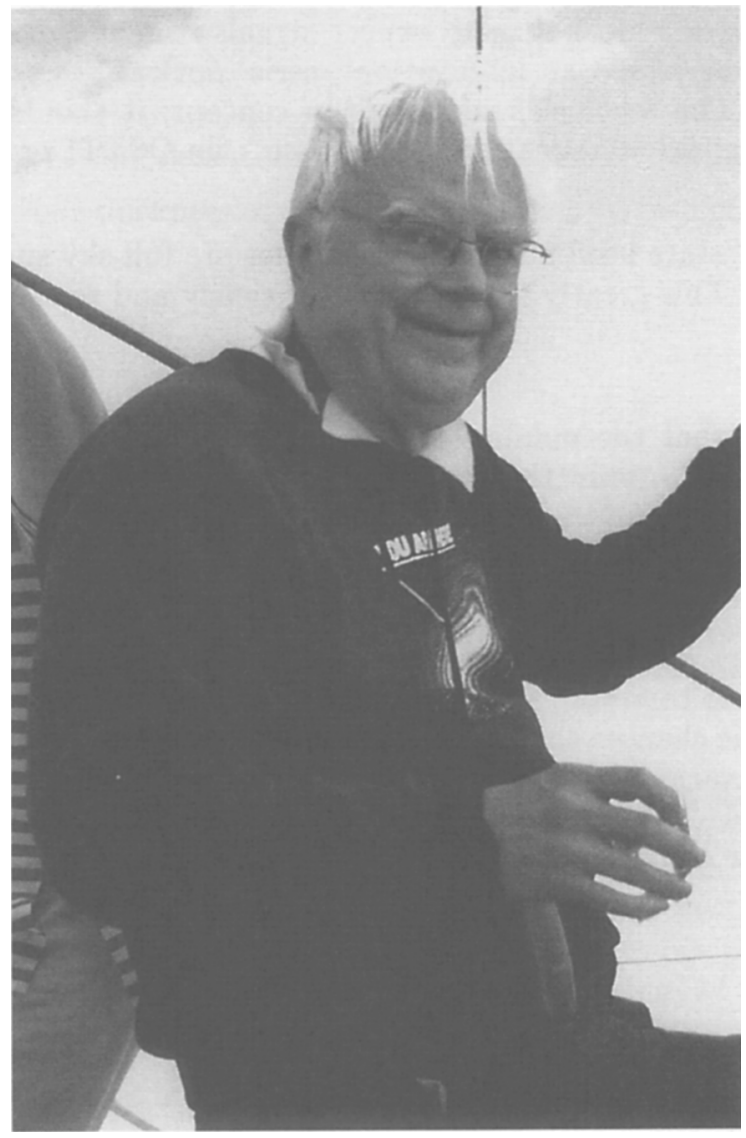

Frank Drake (photo: Seth Shostak) 